\title{
Monotone Iterative Technique for Conformable Fractional Differential Equations with Deviating Arguments
}

\author{
Huiling Chen, ${ }^{1}$ Shuman Meng $\mathbb{D}^{1}{ }^{1}$ and Yujun Cui $\mathbb{D}^{2}$ \\ ${ }^{1}$ Department of Applied Mathematics, Shandong University of Science and Technology, Qingdao 266590, China \\ ${ }^{2}$ State Key Laboratory of Mining Disaster Prevention and Control Co-Founded by Shandong Province and the Ministry of Science \\ and Technology, Shandong University of Science and Technology, Qingdao 266590, China
}

Correspondence should be addressed to Yujun Cui; cyj720201@163.com

Received 21 February 2020; Revised 5 April 2020; Accepted 22 September 2020; Published 15 October 2020

Academic Editor: Qasem M. Al-Mdallal

Copyright (c) 2020 Huiling Chen et al. This is an open access article distributed under the Creative Commons Attribution License, which permits unrestricted use, distribution, and reproduction in any medium, provided the original work is properly cited.

\begin{abstract}
This paper is concerned with the existence of extremal solutions for periodic boundary value problems for conformable fractional differential equations with deviating arguments. We first build two comparison principles for the corresponding linear equation with deviating arguments. With the help of new comparison principles, some sufficient conditions for the existence of extremal solutions are established by combining the method of lower and upper solutions and the monotone iterative technique. As an application, an example is presented to enrich the main results of this article.
\end{abstract}

\section{Introduction}

In recent years, people have been paying attention to the progress of the fractional differential equations. In fact, it is the generalization of the ordinary differential equations to a noninteger order. Significantly, fractional differential equations appear more frequently in different fields of science and engineering, such as viscoelasticity, circuit, and neuron modeling [1-3]. Gradually, fractional differential equations are increasingly regarded as effective assistants. We have observed that many papers are exploring the existence of solutions of boundary value problems for fractional differential equations by using nonlinear functional analysis methods such as fixed point theorems, fixed point index on cone, variational methods and critical point theory, the theory of Mawhin coincidence degree, and the upper and lower solution method; see the monographs of Kilbas et al. [1], Podlubny [2], Diethem [3], the papers [4-26], and the references therein. Among them, the monotone iterative technique is an ingenious and effective method that offers theoretical, as well constructive existence results for nonlinear problems via linear iterates $[9-15,17,23,26]$. It yields monotone sequences that converge to the extremal solutions in a sector generated by the upper and lower solutions. For example, the authors of [22] adopted the method of monotone iteration combined with the method of upper and lower solutions to consider the following system of nonlinear fractional differential equations:

$$
\begin{cases}D^{\alpha} v(t)=f(t, v(t), w(t)), & t \in(0, T], \\ D^{\alpha} w(t)=g(t, w(t), v(t)), & t \in(0, T], \\ \left.t^{1-\alpha} v(t)\right|_{t=0}=x_{0}, & \\ \left.t^{1-\alpha} w(t)\right|_{t=0}=y_{0}, & \end{cases}
$$

where $0<T<\infty, f, g \in C([0, T] \times \mathbb{R} \times \mathbb{R}, \mathbb{R}), x_{0}, y_{0} \in \mathbb{R}$, and $x_{0} \leq y_{0}$. In addition, $[15,24]$ used these methods to study the initial value problems for nonlinear fractional differential equations with no deviating arguments. On the basis of [22], Jian et al. [13] successfully investigated the following nonlinear fractional order differential systems with deviating arguments: 


$$
\begin{cases}D^{\alpha} v(t)=f(t, v(t), v(\theta(t)), w(t), w(\theta(t))), & t \in(0,1], \\ D^{\alpha} w(t)=g(t, w(t), w(\theta(t)), v(t), v(\theta(t))), & t \in(0,1], \\ \left.t^{1-\alpha} v(t)\right|_{t=0}=x_{0}, & \\ \left.t^{1-\alpha} w(t)\right|_{t=0}=y_{0}, & \end{cases}
$$

where $\theta \in C([0,1],[0,1])$. They introduce two well-defined monotone sequences that converge to the solution of the system and, then, establish the existence and uniqueness of the solution of the system. Finally, a numerical iterative scheme is introduced to obtain an accurate approximate solution for the systems.

Motivated by the abovementioned papers, in this paper, we devote ourselves to the existence of solutions to the following boundary value problems with deviation arguments:

$$
\left\{\begin{array}{l}
\mathscr{D}^{\delta} \phi(t)=f(t, \phi(t), \phi(\theta(t))), \quad t \in[0, T], \\
\phi(0)=\phi(T),
\end{array}\right.
$$

where $\delta \in 0,1], \theta \in C([0,1],[0,1])$, and $f \in C([0,1] \times \mathbb{R} \times$ $\mathbb{R}, \mathbb{R})$, and $\mathscr{D}^{\delta} \phi$ is the conformable fractional derivative of order $\delta$. The conformable fractional calculus which was introduced in the work of Khalil et al. [27], then developed by Abdeljawad [28], have been receiving a lot of attention due to the wide application in physics and engineering $[29,30]$. The reader is referred to $[14,16,17,27-33]$ and references therein for some recent advances in conformable fractional calculus and its applications.

In this paper, by establishing two comparison results and using the monotone iterative technique combined with the method of upper and lower solutions, some sufficient conditions are presented for the existence of extremal solutions for periodic boundary value problem (3).

\section{Preliminaries}

Definition 1 (See [27]). Let $f:[0,+\infty \longrightarrow \mathbb{R}$ and $t>0$, and the conformable fractional derivative of order $0<\alpha \leq 1$ is defined by

$$
D_{\alpha} f(t)=\lim _{\rho \longrightarrow 0} \frac{f\left(t+\rho t^{1-\alpha}\right)-f(t)}{\rho},
$$

for $t>0$, and the conformable fractional derivative at 0 is defined as $D_{\alpha} f(0)=\lim \left(D_{\alpha} f\right)(t)$. If $f$ is differentiable, then $D_{\alpha} f(t)=t^{1-\alpha} f^{\prime t}(t)$.

Definition 2 (See [27]). Let $\alpha \in 0,1]$. The conformable fractional integral of a function $f:[0,+\infty \longrightarrow \mathbb{R}$ of order $\alpha$ is denoted as

$$
I_{\alpha} f(t)=\int_{0}^{t} s^{\alpha-1} f(s) \mathrm{d} s
$$

Lemma 1 (See [32]). Let $T>0$. Assume that $f \in C[0, T]$ and $D_{\alpha} f \in C(0, T) \cap L(0, T)$ with $0<\alpha \leq 1$. Then, we have

$$
I_{\alpha} D_{\alpha} f(t)=f(t)-f(0)
$$

Lemma 2 (See [27]). Let $\alpha \in 0,1, l_{1}, l_{2}, q, K \in \mathbb{R}$, and the functions $f, h$ be $\alpha$-differentiable on $[0,+\infty)$. Then,

(a) $D_{\alpha} K=0$ for all constant functions $f(t)=K$

(b) $D_{\alpha}\left(l_{1} f+l_{2} f\right)=l_{1} D_{\alpha} f(t)+l_{2} D_{\alpha} h(t)$

(c) $D_{\alpha} t^{q}=q t^{q-\alpha}$

(d) $D_{\alpha}(f h)=f(t) D_{\alpha} h(t)+h(t) D_{\alpha} f(t)$

(e) $D_{\alpha}(f / h)=\left(\left(h D_{\alpha} f-f D_{\alpha} h\right) / h^{2}\right)$ when $h(t) \neq 0$

Lemma 3 (See [34]). Let $\mathscr{A}: X \longrightarrow X$ linear operator, $r(\mathscr{A})$ be the spectral radius of $\mathscr{A}$, and $\|\mathscr{A}\|=\max _{\|\phi\|=1}\|\mathscr{A} \phi\|$. Then,

(1) $r(\mathscr{A}) \leq\|\mathscr{A}\|$

(2) if $r(\mathscr{A})<1$, then $(\mathscr{I}-\mathscr{A})^{-1}$ exists and $(\mathscr{I}-\mathscr{A})^{-1}=\sum_{n=0}^{\infty} \mathscr{A}^{n}$, where $\mathscr{I}$ stands for the identity operator

It is given that $T>0$. Let $E=C[0, T]$; then, $E$ is a Banach space with the norm $\|x\|=\max _{t \in[0, T]}|x(t)|$.

Let us introduce the following values and functions which will be used in the rest paper.

$$
\begin{aligned}
& K_{1}=\frac{K}{\delta}, \\
& l=e^{-K_{1} T^{\delta}}, \\
& M=\frac{e^{K_{1} T^{\delta}}}{e^{K_{1} T^{\delta}}-1}, \\
& \Psi_{1}(t) \equiv M l, \\
& \Psi_{2}(t) \equiv M, \\
& t \in[0, T], \\
& \bar{M}=M l+\frac{\delta N^{2} M^{2} l^{2} T^{\delta}}{K\left(\delta^{2}-N^{2} M^{2} l^{2} T^{2 \delta}\right)} \\
& -\frac{\delta^{2} N M}{K\left(\delta^{2}-N^{2} M^{2} T^{2 \delta}\right)} \\
& \tilde{M}=M l-\frac{M^{2} N T^{\delta}}{\delta}+\frac{\delta N^{2} M^{2} l^{2} T^{\delta}}{K\left(\delta^{2}-N^{2} M^{2} l^{2} T^{2 \delta}\right)} \\
& -\frac{N^{3} M^{3} T^{2 \delta}}{K\left(\delta^{2}-N^{2} M^{2} T^{2 \delta}\right)}
\end{aligned}
$$

For the forthcoming analysis, we first consider the following two boundary value problems for a linear differential fractional equations: 


$$
\begin{gathered}
\left\{\begin{array}{l}
\mathscr{D}^{\delta} \phi(t)+K \phi(t)=h(t), \quad t \in[0, T], \\
\phi(0)=\phi(T)+a,
\end{array}\right. \\
\left\{\begin{array}{l}
\mathscr{D}^{\delta} \phi(t)+K \phi(t)+N \phi(\theta(t))=h(t), \quad t \in[0, T], \\
\phi(0)=\phi(T)+a .
\end{array}\right.
\end{gathered}
$$

Lemma 4. Let $K>0, a \in \mathbb{R}$, and $h \in E$. Then, problem (8) has the unique solution:

$$
\phi(t)=\int_{0}^{T} G(t, s) h(s) \mathrm{d} s+a \Psi(t),
$$

where $\Psi(t)=\left(1 /\left(1-e^{-K_{1} T^{\delta}}\right)\right) e^{-K_{1} t^{\delta}}$ and

$$
G(t, s)= \begin{cases}\frac{e^{K_{1} T^{\delta}}}{e^{K_{1} T^{\delta}}-1} e^{-K_{1}\left(t^{\delta}-s^{\delta}\right)} s^{\delta-1}, & 0<s \leq t \leq T, \\ \frac{1}{e^{K_{1} T^{\delta}}-1} e^{-K_{1}\left(t^{\delta}-s^{\delta}\right)} s^{\delta-1}, & 0 \leq t<s \leq T .\end{cases}
$$

Proof. Multiply both sides of the first equation of (8) by $e^{K_{1} t^{\circ}}$, namely,

$$
e^{K_{1} t^{\delta}} D^{\delta} \phi(t)+K e^{K_{1} t^{\delta}} \phi(t)=e^{K_{1} t^{\delta}} h(t) .
$$

By using Lemma 2 (d), equation (12) is equivalent to

$$
\mathscr{D}^{\delta}\left[e^{K_{1} t^{\delta}} \phi(t)\right]=e^{K_{1} t^{\delta}} h(t) .
$$

In view of Lemma 1 and Definition 2, we get

$$
e^{K_{1} t^{\delta}} \phi(t)-\phi(0)=\int_{0}^{t} s^{\delta-1} e^{K_{1} s^{\delta}} h(s) \mathrm{d} s
$$

so

$$
\phi(t)=e^{-K_{1} t^{\delta}}\left[\phi(0)+\int_{0}^{t} s^{\delta-1} e^{K_{1} s^{\delta}} h(s) \mathrm{d} s\right] .
$$

The boundary condition $\phi(0)=\phi(T)+a$ leads to

$\phi(0)=\phi(T)+a=e^{-K_{1} T^{\delta}}\left[\phi(0)+\int_{0}^{T} s^{\delta-1} e^{K_{1} s^{\delta}} h(s) \mathrm{d} s\right]+a$.

Clearly,

$$
\phi(0)=\frac{1}{e^{K_{1} T^{\delta}}-1} \int_{0}^{T} s^{\delta-1} e^{K_{1} s^{\delta}} h(s) \mathrm{d} s+\frac{a}{1-e^{-K_{1} T^{\delta}}} .
$$

Substituting (17) into (15), it follows that linear problem (8) has the following integral representation of the solution:

$$
\begin{aligned}
\phi(t)= & e^{-K_{1} t^{\delta}}\left[\frac{1}{e^{K_{1} T^{\delta}}-1} \int_{0}^{T} s^{\delta-1} e^{K_{1} s^{\delta}} h(s) \mathrm{d} s+\int_{0}^{t} s^{\delta-1} e^{K_{1} s^{\delta}} h(s) \mathrm{d} s\right] \\
& +\frac{a}{1-e^{-K_{1} T^{\delta}}} e^{-K_{1} t^{\delta}}, \\
= & \frac{e^{K_{1} T^{\delta}}}{e^{K_{1} T^{\delta}}-1} \int_{0}^{t} s^{\delta-1} e^{-K_{1}\left(t^{\delta}-s^{\delta}\right)} h(s) \mathrm{d} s \\
& +\frac{1}{e^{K_{1} T^{\delta}}-1} \int_{t}^{T} s^{\delta-1} e^{-K_{1}\left(t^{\delta}-s^{\delta}\right)} h(s) \mathrm{d} s+\frac{a}{1-e^{-K_{1} T^{\delta}}} e^{-K_{1} t^{\delta}}, \\
= & \int_{0}^{T} G(t, s) h(s) \mathrm{d} s+a \Psi(t) .
\end{aligned}
$$

This completes the proof.

For all $0<\delta \leq 1$, Green's function $G$ admits the following properties:

$\frac{1}{e^{K_{1} T^{\delta}}-1} s^{\delta-1} \leq G(t, s) \leq \frac{e^{K_{1} T^{\delta}}}{e^{K_{1} T^{\delta}}-1} s^{\delta-1}, \quad t \in[0, T], s \in(0, T]$.

Namely,

$M l s^{\delta-1} \leq G(t, s) \leq M s^{\delta-1}, \quad t \in[0, T], s \in(0, T]$.

In addition, for $\Psi$ given in Lemma 4 , we can get

$$
\Psi_{1}(t)=\frac{e^{-K_{1} T^{\delta}}}{1-e^{-K_{1} T^{\delta}}} \leq \Psi(t) \leq \frac{1}{1-e^{-K_{1} T^{\delta}}}=\Psi_{2}(t) .
$$

We define the operator $\mathscr{A}$ on $E$ by

$$
(\mathscr{A} h)(t)=\int_{0}^{T} G(t, s) h(s) \mathrm{d} s, \quad h \in E .
$$

It is easy to see that $\mathscr{A}: E \longrightarrow E$ is a positive linear continuous operator.

Lemma 5. $\|\mathscr{A}\|=(1 / K)$.

Proof. By direct computation, one has

$$
\int_{0}^{T} G(t, s) \mathrm{d} s=\frac{e^{K_{1} T^{\delta}}}{e^{K_{1} T^{\delta}}-1} \int_{0}^{t} s^{\delta-1} e^{-K_{1}\left(t^{\delta}-s^{\delta}\right)} \mathrm{d} s+\frac{1}{e^{K_{1} T^{\delta}}-1}
$$

$$
\int_{t}^{T} s^{\delta-1} e^{-K_{1}\left(t^{\delta}-s^{\delta}\right)} \mathrm{d} s
$$$$
=\frac{e^{K_{1} T^{\delta}}}{\delta K_{1}\left(e^{K_{1} T^{\delta}}-1\right)}\left(1-e^{-K_{1} t^{\delta}}\right)+\frac{1}{\delta K_{1}\left(e^{K_{1} T^{\delta}}-1\right)}
$$$$
\left(e^{K_{1} T^{\delta}-K_{1} t^{\delta}}-1\right)
$$

$=\frac{1}{\delta K_{1}}=\frac{1}{K}$.

Then, for any $h \in E$, we have 
$\|\mathscr{A} h\|=\max _{t \in[0, T]}|(\mathscr{A} h)(t)| \leq \max _{t \in[0, T]} \int_{0}^{T} G(t, s) \mathrm{d} s \cdot\|h\|=\frac{1}{K}\|h\|$,

which implies that $\|\mathscr{A}\| \leq(1 / K)$. On the other hand, take $h_{0}(t) \equiv 1$, then $h_{0} \in E,\left\|h_{0}\right\|=1$, and

$$
\left\|\mathscr{A} h_{0}\right\|=\max _{t \in[0, T]}|(\mathscr{A} h)(t)|=\int_{0}^{T} G(t, s) \mathrm{d} s=\frac{1}{K}\left\|h_{0}\right\| .
$$

This yields $\|\mathscr{A}\| \geq(1 / K)$. Therefore, $\|\mathscr{A}\|=(1 / K)$. This completes the proof.

We recall that $l=e^{-K_{1} T^{\delta}}$. Then, $l \in(0,1)$. For $\forall h \in C([0, T],[0,+\infty))$, it follows from $(20)$ that

$$
\begin{aligned}
& (\mathscr{A} h)(t)=\int_{0}^{T} G(t, s) h(s) \mathrm{d} s \leq M \int_{0}^{T} s^{\delta-1} h(s) \mathrm{d} s, \quad t \in[0, T], \\
& (\mathscr{A} h)(t)=\int_{0}^{T} G(t, s) h(s) \mathrm{d} s \geq M l \int_{0}^{T} s^{\delta-1} h(s) \mathrm{d} s, \quad t \in[0, T] .
\end{aligned}
$$

The abovementioned two inequalities show that $(\mathscr{A} h)(t) \geq l(\mathscr{A} h)(s), \quad \forall t, s \in[0, T], \forall h \in C([0, T),[0,+\infty)]$. on (9).

Based on the above analysis, we have the following result

Lemma 6. Let $K>0,0 \leq N<K, a \in \mathbb{R}, \theta \in C([0, T],[0, T])$, and $h \in E$. Then, problem (9) has a unique solution.

Proof. From Lemma 4, it follows that $\phi \in E$ is a solution of (9) if and only if

$$
\phi(t)=\int_{0}^{1} G(t, s)[-N \phi(\theta(s))+h(s)] \mathrm{d} s+a \Psi(t) .
$$

Now, we introduce an operator $\mathscr{B}: E \longrightarrow E$ as follows:

$$
(\mathscr{B} \phi)(t)=N \phi(\theta(t)), \quad t \in[0, T] .
$$

It is easy to see that $\mathscr{B}$ is a positive linear operator with $\|\mathscr{B}\|=N$. Thus, (28) reduces to

$$
(I+\mathscr{A} \mathscr{B}) \phi(t)=\mathscr{A} h(t)+a \Psi(t) .
$$

Note from Lemma 5 that $\|\mathscr{A} \mathscr{B}\| \leq\|\mathscr{A}\| \cdot\|\mathscr{B}\|=(\mathrm{N} /$ $K)<1$. Thus, it follows from Lemma 3 that $(I+\mathscr{A} \mathscr{B})^{-1}$ exists and

$$
\begin{aligned}
(I+\mathscr{A} \mathscr{B})^{-1}=\sum_{i=0}^{\infty}(-1)^{i}(\mathscr{A} \mathscr{B})^{i}=I-\mathscr{A} \mathscr{B}+(\mathscr{A} \mathscr{B})^{2}+\cdots \\
+(-1)^{n}(\mathscr{A} \mathscr{B})^{n}+\cdots
\end{aligned}
$$

Therefore, the unique solution of (9) is given by

$$
\phi(t)=\sum_{i=0}^{\infty}(-1)^{i}(\mathscr{A} \mathscr{B})^{i} \mathscr{A} h(t)+a \sum_{i=0}^{\infty}(-1)^{i}(\mathscr{A} \mathscr{B})^{i} \Psi(t) .
$$

The proof is complete.

Now, we present two comparison results.

Lemma 7. Let $K>0, \quad 0 \leq N \leq K l^{2}, \quad a \in \mathbb{R}$, and $\theta \in C([0, T],[0, T])$. Assume that $\phi \in E$ satisfies $\mathscr{D}^{\delta} \phi \in E$ and

$$
\left\{\begin{array}{l}
\mathscr{D}^{\delta} \phi(t) \leq-K \phi(t)-N \phi(\theta(t)), \quad t \in[0, T], \\
\phi(0) \leq \phi(T) .
\end{array}\right.
$$

Then, $\phi(t) \leq 0$ for all $t \in[0, T]$.

Proof. Take $h(t)=\mathscr{D}^{\delta} \phi(t)+K \phi(t)+N \phi(\theta(t)), a=\phi(0)-$ $\phi(T)$. Then,

$$
\begin{aligned}
h(t) & \leq 0, \\
a & \leq 0 .
\end{aligned}
$$

Applying Lemma 6, (32) holds, and (32) can be expressed by

$$
\phi(t)=\sum_{i=0}^{\infty}(\mathscr{A} \mathscr{B})^{2 i}(I-\mathscr{A} \mathscr{B}) \mathscr{A} h(t)+a \sum_{i=0}^{\infty}(\mathscr{A} \mathscr{B})^{2 i}(I-\mathscr{A} \mathscr{B}) \Psi(t) .
$$

Since $h \leq 0$, it implies that $h_{0}(t) \equiv-(\mathscr{A} h)(0) \geq 0$. Thus, from (27), we obtain

$$
\begin{aligned}
& -\mathscr{A} h \geq l h_{0}, \\
& -\mathscr{A} h \leq \frac{1}{l} h_{0} .
\end{aligned}
$$

With the help of positivity of operator $\mathscr{A} \mathscr{B}$, the definition of operator $\mathscr{B}$, and (23), we have

$$
-(\mathscr{A} \mathscr{B}) \mathscr{A} h \leq \frac{1}{l}(\mathscr{A} \mathscr{B}) h_{0}=\frac{N}{l K} h_{0} .
$$

Consequently, we conclude that

$$
(I-\mathscr{A} \mathscr{B}) \mathscr{A} h \leq-l h_{0}+\frac{N}{l K} h_{0}=-\left(l-\frac{N}{l K}\right) h_{0} \leq 0 .
$$

On the other hand, by (21), we infer that

$$
\begin{aligned}
(I-\mathscr{A} \mathscr{B}) \Psi(t) & =\frac{e^{-K_{1} t^{\delta}}}{1-e^{-K_{1} T^{\delta}}}-N \int_{0}^{T} G(t, s) \frac{e^{-K_{1}(\theta(s))^{\delta}}}{1-e^{-K_{1} T^{\delta}}} \mathrm{d} s \\
& \geq \frac{e^{-K_{1} T^{\delta}}}{1-e^{-K_{1} T^{\delta}}}-\frac{N}{1-e^{-K_{1} T^{\delta}}} \int_{0}^{T} G(t, s) \mathrm{d} s, \\
& =\frac{1}{1-e^{-K_{1} T^{\delta}}}\left(l-\frac{N}{K}\right) \geq 0 .
\end{aligned}
$$

Hence, $\phi(t) \leq 0$ holds for all $t \in[0, T]$ that follow from $a \leq 0$ and (35). This completes the proof.

Lemma 8. Let $K>0, \quad 0 \leq N M T^{\delta}<\delta, \quad 0 \leq N<K, \quad \tilde{M}>0$, $\bar{M}>0$, and $\theta \in C([0, T],[0, T])$. Assume that $\phi \in E$ satisfies $\mathscr{D}^{\delta} \phi \in E$ and (33). Then, $\phi(t) \leq 0$ for all $t \in[0, T]$. 
Proof. Take again $h(t)=\mathscr{D}^{\delta} \phi(t)+K \phi(t)+N \phi(\theta(t))$, $a=\phi(0)-\phi(T)$. Then,

$$
\begin{array}{r}
h(t) \leq 0, \\
a \leq 0 .
\end{array}
$$

Applying Lemma 6, (32) holds, and (32) can be expressed by

$$
\begin{aligned}
\phi(t)= & \sum_{i=0}^{\infty}(\mathscr{A} \mathscr{B})^{2 i} \mathscr{A} h(t)-\sum_{i=0}^{\infty}(\mathscr{A} \mathscr{B})^{2 i+1} \mathscr{A} h(t) \\
& +a\left[\sum_{i=0}^{\infty}(\mathscr{A} \mathscr{B})^{i} \Psi(t)-\sum_{i=0}^{\infty}(\mathscr{A} \mathscr{B})^{2 i+1} \Psi(t)\right] .
\end{aligned}
$$

Taking notice of the fact that $h(t) \leq 0$, by (20), we have

$$
(\mathscr{A} h)(t)=\int_{0}^{T} G(t, s) h(s) \mathrm{d} s \leq M l \int_{0}^{T} s^{\delta-1} h(s) \mathrm{d} s,
$$

and for $n \geq 1$,

$$
\begin{aligned}
(\mathscr{A} \mathscr{B})^{2 n}(\mathscr{A} h)(t) & =N^{2 n} \int_{0}^{T} G(t, s) \int_{0}^{T} G\left(\theta(s), \tau_{2 n-1}\right) \\
& \int_{0}^{T} G\left(\theta\left(\tau_{2 n-1}\right), \tau_{2 n-2}\right) \cdots \int_{0}^{T} G\left(\theta\left(\tau_{2}\right), \tau_{1}\right) \\
& \int_{0}^{T} G\left(\theta\left(\tau_{1}\right), \tau_{0}\right) h\left(\tau_{0}\right) d \tau_{0} d \tau_{1}, \ldots, d \tau_{2 n-1} \mathrm{~d} s \\
\leq & N^{2 n} \int_{0}^{T} G(t, s) \mathrm{d} s\left(M l \int_{0}^{T} s^{\delta-1} \mathrm{~d} s\right)^{2 n-1} \\
& \cdot \int_{0}^{T} M l \tau_{0}^{\delta-1} h\left(\tau_{0}\right) \mathrm{d} \tau_{0}, \\
= & \frac{N^{2 n} M^{2 n} l^{2 n}}{K}\left(\frac{T^{\delta}}{\delta}\right)^{2 n-1} \int_{0}^{T} \tau_{0}^{\delta-1} h\left(\tau_{0}\right) \mathrm{d} \tau_{0},
\end{aligned}
$$

and for $n \geq 1$,

$$
\begin{aligned}
(\mathscr{A} \mathscr{B})^{2 n+1}(\mathscr{A} h)(t) & =N^{2 n+1} \int_{0}^{T} G(t, s) \int_{0}^{T} G\left(\theta(s), \tau_{2 n}\right) \\
& \int_{0}^{T} G\left(\theta\left(\tau_{2 n}\right), \tau_{2 n-1}\right) \ldots \int_{0}^{T} G\left(\theta\left(\tau_{2}\right), \tau_{1}\right) \\
& \int_{0}^{T} G\left(\theta\left(\tau_{1}\right), \tau_{0}\right) h\left(\tau_{0}\right) d \tau_{0} d \tau_{1}, \ldots, d \tau_{2 n} \mathrm{~d} s \\
\leq & N^{2 n+1} \int_{0}^{T} G(t, s) \mathrm{d} s\left(M l \int_{0}^{T} s^{\delta-1} \mathrm{~d} s\right)^{2 n} \\
& \cdot \int_{0}^{T} M l \tau_{0}^{\delta-1} h\left(\tau_{0}\right) \mathrm{d} \tau_{0}, \\
= & \frac{N^{2 n+1} M^{2 n+1}}{K}\left(\frac{T^{\delta}}{\delta}\right)^{2 n} \int_{0}^{T} \tau_{0}^{\delta-1} h\left(\tau_{0}\right) \mathrm{d} \tau_{0} .
\end{aligned}
$$

These lead us to

$$
\begin{aligned}
& \sum_{i=0}^{\infty}(\mathscr{A} \mathscr{B})^{2 i} \mathscr{A} h(t)-\sum_{i=0}^{\infty}(\mathscr{A} \mathscr{B})^{2 i+1} \mathscr{A} h(t) \\
& \leq\left[M l+\sum_{i=1}^{\infty} \frac{N^{2 i} M^{2 i} l^{2 i}}{K}\left(\frac{T^{\delta}}{\delta}\right)^{2 i-1}-\sum_{i=0}^{\infty} \frac{N^{2 i+1} M^{2 i+1}}{K}\left(\frac{T^{\delta}}{\delta}\right)^{2 i}\right] \\
& \int_{0}^{T} s^{\delta-1} h(s) \mathrm{d} s=\bar{M} \int_{0}^{T} s^{\delta-1} h(s) \mathrm{d} s \leq 0 .
\end{aligned}
$$

By (20)-(23) and the positivity of operator $\mathscr{A} \mathscr{B}$, we have

$$
\Psi(t) \geq \frac{e^{-K_{1} T^{\delta}}}{1-e^{-K_{1} T^{\delta}}}=M l,
$$

$$
\begin{aligned}
(\mathscr{A} \mathscr{B}) \Psi(t) & \leq(\mathscr{A} \mathscr{B}) \Psi_{2}(t)=N \int_{0}^{T} G(t, s) \Psi_{2}(\theta(s)) \mathrm{d} s \\
& \leq M^{2} N \int_{0}^{T} s^{\delta-1} \mathrm{~d} s=\frac{M^{2} N T^{\delta}}{\delta}
\end{aligned}
$$

and for $n \geq 1$,

$$
\begin{aligned}
(\mathscr{A} \mathscr{B})^{2 n} \Psi(t) \geq & (\mathscr{A} \mathscr{B})^{2 n} \Psi_{1}(t) \\
= & N^{2 n} \int_{0}^{T} G(t, s) \int_{0}^{T} G\left(\theta(s), \tau_{2 n-1}\right) \\
& \cdot \int_{0}^{T} G\left(\theta\left(\tau_{2 n-1}\right), \tau_{2 n-2}\right) \\
& \ldots \int_{0}^{T} G\left(\theta\left(\tau_{2}\right), \tau_{1}\right) \Psi_{1}\left(\theta\left(\tau_{1}\right)\right) d \tau_{1} d \tau_{2}, \ldots, d \tau_{2 n-1} \mathrm{~d} s \\
\geq & N^{2 n} \Psi_{1}(t) \int_{0}^{T} G(t, s) \mathrm{d} s\left(\int_{0}^{T} M l s^{\delta-1} \mathrm{~d} s\right)^{2 n-1}, \\
= & \frac{N^{2 n}}{K} M l\left(\frac{M l T^{\delta}}{\delta}\right)^{2 n-1}=\frac{N^{2 n} M^{2 n} l^{2 n} T^{(2 n-1) \delta}}{K \delta^{2 n-1}},
\end{aligned}
$$

and for $n \geq 1$,

$$
\begin{aligned}
(\mathscr{A} \mathscr{B})^{2 n+1} \Psi(t) \leq & (\mathscr{A} \mathscr{B})^{2 n+1} \Psi_{2}(t) \\
= & N^{2 n+1} \int_{0}^{T} G(t, s) \int_{0}^{T} G\left(\theta(s), \tau_{2 n}\right) \\
& \cdot \int_{0}^{T} G\left(\theta\left(\tau_{2 n}\right), \tau_{2 n-1}\right) \\
& \ldots \int_{0}^{T} G\left(\theta\left(\tau_{2}\right), \tau_{1}\right) \Psi_{2}\left(\theta\left(\tau_{1}\right)\right) d \tau_{1} d \tau_{2}, \ldots, d \tau_{2 n} \mathrm{~d} s \\
\leq & N^{2 n+1} \Psi_{2}(t) \int_{0}^{T} G(t, s) \mathrm{d} s\left(\int_{0}^{T} M l s^{\delta-1} \mathrm{~d} s\right)^{2}, \\
= & \frac{N^{2 n+1}}{K} M l\left(\frac{M T^{\delta}}{\delta}\right)^{2 n}=\frac{N^{2 n+1} M^{2 n+1}}{K \delta^{2 n}} T^{2 n \delta} .
\end{aligned}
$$

These, together with the fact that $a \leq 0$, ensure that 


$$
\begin{aligned}
& a\left[\sum_{i=0}^{\infty}(\mathscr{A} \mathscr{B})^{i} \Psi(t)-\sum_{i=0}^{\infty}(\mathscr{A} \mathscr{B})^{2 i+1} \Psi(t)\right] \\
& =a\left[\Psi(t)-(\mathscr{A} \mathscr{B}) \Psi(t)+\sum_{i=1}^{\infty}(\mathscr{A} \mathscr{B})^{i} \Psi(t)-\sum_{i=1}^{\infty}(\mathscr{A} \mathscr{B})^{2 i+1} \Psi(t)\right] \\
& \leq a\left[M l-\frac{M^{2} N T^{\delta}}{\delta}+\sum_{i=1}^{\infty} \frac{N^{2 i} M^{2 i} l^{2 i} T^{(2 i-1) \delta}}{K \delta^{2 i-1}}-\sum_{i=1}^{\infty} \frac{N^{2 i+1} M^{2 i+1}}{K \delta^{2 i}} T^{2 i \delta}\right], \\
& =a \tilde{M} \leq 0 .
\end{aligned}
$$

Thus, by (41), (45), and (49), we have that $\phi(t) \leq 0$ for all $t \in[0, T]$, and the lemma is proved.

\section{Main Results}

Now, we are in the position to prove the existence of extremal solutions of (3) by using the monotone iterative method of lower and upper solutions. To this end, we define the lower and upper solutions of (3).

Definition 3. A function $u_{0} \in E$ satisfying $\mathscr{D}^{\delta} u_{0} \in E$ is called a lower solution of problem (3) if it satisfies

$$
\left\{\begin{array}{l}
\mathscr{D}^{\delta} u_{0}(t) \leq f\left(t, u_{0}(t), u_{0}(\theta(t))\right), \quad t \in[0, T], \\
u_{0}(0) \leq u_{0}(T) .
\end{array}\right.
$$

Analogously, a function $w_{0} \in E$ satisfying $\mathscr{D}^{\delta} w_{0} \in E$ is called an upper solution of (3) if the inequalities

$$
\left\{\begin{array}{l}
\mathscr{D}^{\delta} w_{0}(t) \geq f\left(t, w_{0}(t), w_{0}(\theta(t))\right), \quad t \in[0, T] \\
w_{0}(0) \geq w_{0}(T)
\end{array}\right.
$$

hold.

Theorem 1. Assume that the following conditions hold: $\left(H_{1}\right) \theta \in C([0, T],[0, T])$

$\left(H_{2}\right)$ : the functions $u_{0}$ and $w_{0}$ are lower and upper solutions of problem (3), respectively, such that $u_{0}(t) \leq w_{0}(t)$ on $[0, T]$

$\left(H_{3}\right) f \in C\left([0, T] \times \mathbb{R}^{2}, \mathbb{R}\right)$ and there exist $K>0, N \geq 0$ such that

$f(t, x, z)-f(t, \bar{x}, \bar{z}) \geq-K(x-\bar{x})-N(z-\bar{z})$,

for all $t \in[0, T], \quad u_{0}(t) \leq \bar{x} \leq x \leq w_{0}(t), \quad u_{0}(t) \leq \bar{z} \leq$ $z \leq w_{0}(t)$

$\left(H_{4}\right)$ : the inequality $N \leq K l^{2}$ holds or the inequalities $N M T^{\delta}<\delta, N<K, \tilde{M}>0, \bar{M}>0$ hold

Then, (3) has minimal and maximal solution $u, w$ in the sector $\left[u_{0}, w_{0}\right]$, which can be obtained by monotone iterative sequences starting from $u_{0}$ and $w_{0}$, where $\left[u_{0}, w_{0}\right]=\left\{z \in E: u_{0}(t) \leq z(t) \leq w_{0}(t), t \in[0, T]\right\}$.

Proof. For $k=1,2, \ldots$, let us define

$$
\begin{aligned}
& \left\{\begin{array}{l}
\mathscr{D}^{\delta} u_{k}(t)+K u_{k}(t)+N u_{k}(\theta(t))=f\left(t, u_{k-1}(t), u_{k-1}(\theta(t))\right)+K u_{k-1}(t)+N u_{k-1}(\theta(t)), \quad t \in[0, T], \\
u_{k}(0)=u_{k}(T),
\end{array}\right. \\
& \left\{\begin{array}{l}
\mathscr{D}^{\delta} w_{k}(t)+K w_{k}(t)+N w_{k}(\theta(t))=f\left(t, w_{k-1}(t), w_{k-1}(\theta(t))\right)+K w_{k-1}(t)+N w_{k-1}(\theta(t)), \quad t \in[0, T], \\
w_{k}(0)=w_{k}(T) .
\end{array}\right.
\end{aligned}
$$

By Lemma 6 , for any $k=1,2, \ldots$, we know that linear problems (53) and (54) have a unique solution $u_{k}(t), w_{k}(t)$, respectively, which implies that the sequences $\left\{u_{k}(t)\right\}$, $\left\{w_{k}(t)\right\}$ are well defined. Furthermore, $u_{k}(t), w_{k}(t)$ can be expressed as

$$
\begin{aligned}
u_{k}(t) & =(I+\mathscr{A} \mathscr{B})^{-1} \mathscr{A} \mathscr{F} u_{k-1}(t), \\
w_{k}(t) & =(I+\mathscr{A} \mathscr{B})^{-1} \mathscr{A} \mathscr{F} w_{k-1}(t),
\end{aligned}
$$

where $\mathscr{F}: E \longrightarrow E$ is a bounded operator defined by

$$
(\mathscr{F} u)(t)=f(t, u(t), u(\theta(t)))+K u(t)+N u(t), \quad u \in E .
$$

By the integral expression of operator $\mathscr{A}$, it is easy to see that $\mathscr{A}$ is completely continuous. Hence, $(I+\mathscr{A} \mathscr{B})^{-1} \mathscr{A} \mathscr{F}$ is completely continuous.

Firstly, let us prove that

$$
u_{0} \leq u_{1} \leq w_{1} \leq w_{0} .
$$

To do this, let $v(t)=u_{0}(t)-u_{1}(t)$. By the definition of the lower solution, we get

$$
\begin{aligned}
\mathscr{D}^{\delta} v(t)= & \mathscr{D}^{\delta} u_{0}(t)-\mathscr{D}^{\delta} u_{1}(t) \\
\leq & f\left(t, u_{0}(t), u_{0}(\theta(t))\right)-f\left(t, u_{0}(t), u_{0}(\theta(t))\right) \\
& +K\left(u_{1}(t)-u_{0}(t)\right) \\
& +N\left(u_{1}(\theta(t))-u_{0}(\theta(t))\right) \\
= & -K v(t)-N v(\theta(t)), \quad t \in[0, T] \\
v(0)= & u_{0}(0)-u_{1}(0) \leq u_{0}(T)-u_{1}(T)=v(T) .
\end{aligned}
$$

This shows, by Lemma 7 or Lemma 8 , that $v(t) \leq 0$ on $[0, T]$, and hence, $u_{0} \leq u_{1}$. Similarly, we can deduce that $w_{1} \leq w_{0}$. 

obtain

Now, let $v(t)=u_{1}(t)-w_{1}(t)$; by $\left(H_{2}\right)$ and $\left(H_{3}\right)$, we

$$
\begin{aligned}
\mathscr{D}^{\delta} v(t)= & \mathscr{D}^{\delta} u_{1}(t)-\mathscr{D}^{\delta} w_{1}(t), \\
= & f\left(t, u_{0}(t), u_{0}(\theta(t))\right)-K\left(u_{1}(t)-u_{0}(t)\right) \\
& -N\left(u_{1}(\theta(t))-u_{0}(\theta(t))\right) \\
& -f\left(t, w_{0}(t), w_{0}(\theta(t))\right)+K\left(w_{1}(t)-w_{0}(t)\right) \\
& +N\left(w_{1}(\theta(t))-w_{0}(\theta(t))\right) \\
\leq & -K\left[u_{0}(t)-w_{0}(t)\right]-N\left[u_{0}(\theta(t))-w_{0}(\theta(t))\right] \\
& -K\left[u_{1}(t)-u_{0}(t)-w_{1}(t)+w_{0}(t)\right] \\
& +N\left[-u_{1}(\theta(t))+u_{0}+w_{1}(\theta(t))-w_{0}(\theta(t))\right], \\
= & -K v(t)-N v(\theta(t)), \quad t \in[0, T], \\
v(0)= & u_{1}(0)-w_{1}(0)=u_{1}(T)-w_{1}(T)=v(T) .
\end{aligned}
$$

Then, from Lemma 7 or Lemma 8, we get $v(t) \leq 0$, which yields $u_{1} \leq w_{1}$.

Secondly, we need to show that $u_{1}$ and $w_{1}$ are the lower and upper solutions of problem (3), respectively. In fact, it follows from $\left(\mathrm{H}_{2}\right)$ and $\left(\mathrm{H}_{3}\right)$ that

$$
\left\{\begin{array}{l}
\mathscr{D}^{\delta} u_{1}(t)=f\left(t, u_{0}(t), u_{0}(\theta(t))\right)-K\left(u_{1}(t)-u_{0}(t)\right)-N\left(u_{1}(\theta(t))-u_{0}(\theta(t))\right) \\
-f\left(t, u_{1}(t), u_{1}(\theta(t))\right)+f\left(t, u_{1}(t), u_{1}(\theta(t))\right) \\
\leq-K\left[u_{0}(t)-u_{1}(t)\right]-N\left[u_{0}(\theta(t))-u_{1}(\theta(t))\right]-K\left(u_{1}(t)-u_{0}(t)\right) \\
-N\left(u_{1}(\theta(t))-u_{0}(\theta(t))\right)+f\left(t, u_{1}(t), u_{1}(\theta(t))\right) \\
=f\left(t, u_{1}(t), u_{1}(\theta(t))\right) \\
u_{1}(0)=u_{1}(T)
\end{array}\right.
$$

which show that $v_{1}$ is a lower solution of problem (3). Similarly, we can conclude that $w_{1}$ is an upper solution of problem (3).

Repeating the foregoing arguments, we can prove that the sequences $\left\{u_{k}(t)\right\},\left\{w_{k}(t)\right\}$ are lower and upper solutions of problem (3), respectively, and satisfy the following inequality:

$$
u_{0} \leq u_{1} \cdots \leq u_{k} \leq \cdots \leq w_{k} \leq \cdots \leq w_{1} \leq w_{0} .
$$

Obviously, the sequences $\left\{u_{k}(t)\right\},\left\{w_{k}(t)\right\}$ are uniformly bounded in $E$ and by (55) and the complete continuity of operator $(I+\mathscr{A} \mathscr{B})^{-1} \mathscr{A} \mathscr{F}$, and it follows that $\left\{u_{k}(t)\right\}$, $\left\{w_{k}(t)\right\}$ are relatively compact. This, together with the monotonicity of the sequences $\left\{u_{k}(t)\right\},\left\{w_{k}(t)\right\}$, guarantees that the sequences $\left\{u_{k}(t)\right\},\left\{w_{k}(t)\right\}$ converge uniformly to $u, w$, respectively, and that $u, w \in\left[v_{0}, w_{0}\right]$ are solutions of (3).

Finally, we prove the minimal and maximal property of $u$ and $w$ on $\left[v_{0}, w_{0}\right]$. We assume that $z \in\left[v_{0}, w_{0}\right]$ is any solution of (3) and there exists a positive integer $k$ such that $u_{k}(t) \leq z(t) \leq w_{k}(t)$ for $t \in[0, T]$.

Let $v(t)=u_{k}(t)-z(t)$, then

$$
\left\{\begin{array}{l}
\mathscr{D}^{\delta} v(t)=\mathscr{D}^{\delta} u_{k}(t)-\mathscr{D}^{\delta} z(t) \\
=f\left(t, u_{k-1}(t), u_{k-1}(\theta(t))\right)-K\left(u_{k}(t)-u_{k-1}(t)\right) \\
-N\left(u_{k}(\theta(t))-u_{k-1}(\theta(t))\right)-f(t, z(t), z(\theta(t))) \\
\leq-K\left[u_{k-1}(t)-z(t)\right]-N\left[u_{k-1}(\theta(t))-z(\theta(t))\right] \\
-K\left(u_{k}(t)-u_{k-1}(t)\right)-N\left(u_{k}(\theta(t))-u_{k-1}(\theta(t))\right) \\
=-K v(t)-N v(\theta(t)) \\
v(0)=u_{k}(0)-z(0)=u_{k}(T)-z(T)=v(T)
\end{array}\right.
$$

undoubtedly, $a(t) \leq 0$, namely, $u_{k}(t) \leq z(t)$. By a similar method, we can show that $z(t) \leq w_{k}(t)$. Thus, $u_{k} \leq z \leq w_{k}$, $k=1,2, \ldots$. It is easy to find that $u(t) \leq z \leq w(t)$ when $k \longrightarrow \infty$. That is $u, w$ are minimal and maximal solutions of (1) in the sector $\left[u_{0}, w_{0}\right]$. The proof is completed.

Then, by applying Lemma 7 or Lemma 8 , we get $v(t) \leq 0$, that is $u_{k}(t) \leq z(t)$ on $[0, T]$. Similarly, we can show that $z(t) \leq w_{k}(t)$ on $[0, T]$. Notice that $u_{0}(t) \leq z(t) \leq w_{0}(t)$ on $[0, T]$. So, $u_{k}(t) \leq z(t) \leq w_{k}(t)$ hold for every $k$ from mathematical induction. Hence, by taking $k \longrightarrow+\infty$, we have $u(t) \leq z(t) \leq w(t)$ on $[0, T]$. The proof is complete.

Example 1. We consider the following BVP: 


$$
\left\{\begin{array}{l}
\mathscr{D}^{(1 / 2)} \phi(t)=-\frac{1}{3(1+\sqrt{2 \pi})^{2}}(1+\phi(t))^{3}+\frac{\sqrt{2}}{60 \pi} \cos \frac{\phi^{2}\left(t^{2}\right)}{4}, \quad t \in[0,1], \\
\phi(0)=\phi(1) .
\end{array}\right.
$$

Obviously, $\delta=(1 / 2), T=1, \theta(t)=t^{2}$, and

$$
f(t, u, v)=-\frac{1}{3(1+\sqrt{2 \pi})^{2}}(1+u)^{3}+\frac{\sqrt{2}}{60 \pi} \cos \frac{v^{2}}{4} \text {. }
$$

$$
\begin{aligned}
& \left\{\begin{array}{l}
\mathscr{D}^{(1 / 2)} u_{0}(t)=0<\frac{\sqrt{2}}{60 \pi} \cos \frac{1}{4}=f\left(t, u_{0}(t), u_{0}(\theta(t))\right), \quad t \in[0,1], \\
u_{0}(0)=u_{0}(1)=-1,
\end{array}\right. \\
& \left\{\begin{array}{l}
\mathscr{D}^{(1 / 2)} w_{0}(t)=0>-\frac{1+\sqrt{2 \pi}}{3}=f\left(t, w_{0}(t), w_{0}(\theta(t))\right), \quad t \in[0,1], \\
w_{0}(0)=w_{0}(1)=\sqrt{2 \pi}
\end{array}\right.
\end{aligned}
$$

This shows that $u_{0}, w_{0}$ are lower and upper solutions of (63). On the other hand, it is easy to verify that $\left(\mathrm{H}_{3}\right)$ holds for $K=1$ and $N=(1 / 60)$. Furthermore, we have

$$
\begin{aligned}
K_{1} & =2, \\
l & =e^{-2} \in(0,1), \\
M & =\frac{1}{1-e^{-2}} \approx 1.1565, \\
N & =\frac{1}{60}<1=K, \\
N M T^{\delta} & \approx 0.0193<\frac{1}{2}=\delta, \\
\bar{M} & \approx 0.1388>0, \\
\tilde{M} & \approx 0.1119>0 .
\end{aligned}
$$

Hence, all conditions of Theorem 1 hold. Therefore, equation (63) has the extremal solution in $\left[v_{0}, w_{0}\right]$.

\section{Data Availability}

The data used to support the findings of this study are available from the corresponding author upon request.

\section{Conflicts of Interest}

The authors declare that they have no conflicts of interest.

\section{Acknowledgments}

This work was supported by the National Natural Science Foundation of China (11571207 and 51774197), the
Shandong Natural Science Foundation (ZR2018MA011), SDUST graduate innovation project (SDKDYC190238), and the Tai'shan Scholar Engineering Construction Fund of Shandong Province of China.

\section{References}

[1] A. A. Kilbas, H. M. Srivastava, and J. J. Trujillo, Theory and Applications of Fractional Differential Equations, Elsevier, Amsterdam, Netherlands, 2006.

[2] I. Podlubny, Fractional Differential Equations, Mathematics in Science and Engineering, Academic Press, New York, NY, USA, 1999.

[3] K. Diethem, The Analysis of Fractional Differential Equations, Springer, Heidelberg, Germany, 2004.

[4] T. Abdeljawad, F. Jarad, S. Mallak, and J. Alzabut, "Lyapunov type inequalities via fractional proportional derivatives and application on the free zero disc of Kilbas-Saigo generalized Mittag-Leffler functions," European Physical Journal Plus, vol. 134, no. 5, Article ID 247, 2019.

[5] A. B. Abdulla, M. Al-Refai, and A. Al-Rawashdeh, "On the existence and uniqueness of solutions for a class of non-linear fractional boundary value problems," Journal of King Saud University-Science, vol. 28, no. 1, pp. 103-110, 2016.

[6] M. A. Alqudah, C. Ravichandran, T. Abdeljawad, and N. Valliammal, "New results on Caputo fractional-order neutral differential inclusions without compactness," Advances in Difference Equations, vol. 2019, no. 1, pp. 1-14, Article ID 528, 2019.

[7] M. Al-Refai and M. A. Hajji, "Monotone iterative sequences for nonlinear boundary value problems of fractional order," Nonlinear Analysis Series A: Theory, Methods and Applications, vol. 74, no. 11, pp. 353-3539, 2011.

[8] M. Al-Refai, "Basic results on nonlinear eigenvalue problems with fractional order," Electronic Journal of Differential Equations, vol. 191, pp. 1-12, 2012. 
[9] Z. Bai, S. Zhang, S. Sun, and C. Yin, "Monotone iterative method for a class of fractional differential equations," Electronic Journal of Differential Equations, vol. 2016, no. 6, pp. 1-8, 2016.

[10] Y. Cui, Q. Sun, and X. Su, "Monotone iterative technique for nonlinear boundary value problems of fractional order $p \in 2,3$," Advances in Difference Equations, vol. 2017, Article ID 248, 2017.

[11] C. Hu, B. Liu, and S. Xie, "Monotone iterative solutions for nonlinear boundary value problems of fractional differential equation with deviating arguments," Applied Mathematics and Computation, vol. 222, pp. 72-81, 2013.

[12] T. Jankowski, "Monotone iterative method for first-order differential equations at resonance," Applied Mathematics and Computation, vol. 233, pp. 20-28, 2014.

[13] H. Jian, B. Liu, and S. Xie, "Monotone iterative solutions for nonlinear fractional differential systems with deviating arguments," Applied Mathematics and Computation, vol. 262, pp. 1-14, 2015.

[14] S. Liu, H. Wang, X. Li, and H. Li, "The extremal iteration solution to a coupled system of nonlinear conformable fractional differential equations," Journal of Nonlinear Sciences and Applications, vol. 10, no. 9, pp. 5082-5089, 2017.

[15] F. A. McRae, "Monotone iterative technique and existence results for fractional differential equations," Nonlinear Analysis: Theory, Methods \& Applications, vol. 71, no. 12, pp. 6093-6096, 2009.

[16] S. Meng and Y. Cui, "Multiplicity results to a conformable fractional differential equations involving integral boundary condition," Complexity, vol. 2019, Article ID 8402347, 8 pages, 2019.

[17] S. Meng and Y. Cui, "The extremal solution to conformable fractional differential equations involving integral boundary condition," Mathematics, vol. 7, Article ID 186, 2019.

[18] C. V. Pao, Nonlinear Parabolic and Elliptic Equations, Plenum Press, New York, NY, USA, 1992.

[19] T. Qi, Y. Liu, and Y. Zou, "Existence result for a class of coupled fractional differential systems with integral boundary value conditions," The Journal of Nonlinear Sciences and Applications, vol. 10, no. 7, pp. 4034-4045, 2017.

[20] M. Syam and M. Al-Refai, "Positive solutions and monotone iterative sequences for a class of higher order boundary value problems of fractional order," Journal of Fractional Calculus and Applications, vol. 4, pp. 1-13, 2013.

[21] S. Song, H. Li, and Y. Zou, "Monotone iterative method for fractional differential equations with integral boundary conditions," Journal of Function Spaces, vol. 2020, Article ID 7319098, 7 pages, 2020.

[22] G. Wang, R. P. Agarwal, and A. Cabada, "Existence results and the monotone iterative technique for systems of nonlinear fractional differential equations," Applied Mathematics Letters, vol. 25, no. 6, pp. 1019-1024, 2012.

[23] G. Wang, "Monotone iterative technique for boundary value problems of a nonlinear fractional differential equation with deviating arguments," Journal of Computational and Applied Mathematics, vol. 236, no. 9, pp. 2425-2430, 2012.

[24] S. Zhang, "Monotone iterative method for initial value problem involving Riemann-Liouville fractional derivatives," Nonlinear Analysis: Theory, Methods \& Applications, vol. 71, no. 5-6, pp. 2087-2093, 2009.

[25] Y. Zou, "Positive solutions for a fractional boundary value problem with a perturbation term," Journal of Function Spaces, vol. 2018, Article ID 9070247, 5 pages, 2018.
[26] G. S. Ladde, V. Lakshmikantham, and A. S. Vatsala, Monotone Iterative Techniques for Nonlinear Differential Equations, Pitman Advanced Publishing Program, London, UK, 1985.

[27] R. Khalil, M. Al Horani, A. Yousef, and M. Sababheh, "A new definition of fractional derivative," Journal of Computational and Applied Mathematics, vol. 264, pp. 65-70, 2014.

[28] T. Abdeljawad, "On conformable fractional calculus," Journal of Computational and Applied Mathematics, vol. 279, pp. 57-66, 2015.

[29] D. Anderson, E. Camrud, and D. Ulness, "On the nature of the conformable derivative and its applications to physics," Journal of Fractional Calculus and Applications, vol. 10, no. 2, pp. 92-135, 2019.

[30] S. Yang, L. Wang, and S. Zhang, "Conformable derivative: application to non-Darcian flow in low-permeability porous media," Applied Mathematics Letters, vol. 79, pp. 105-110, 2018.

[31] O. S. Iyiola and E. R. Nwaeze, "Some new results on the new conformable fractional calculus with application using D'Alambert approach," Progress in Fractional Differentiation and Applications, vol. 2, no. 2, pp. 115-122, 2016.

[32] X. Dong, Z. Bai, and S. Zhang, "Positive solutions to boundary value problems of $p$-Laplacian with fractional derivative," Boundary Value Problems, vol. 2017, p. 5, 2017.

[33] Q. Song, X. Dong, Z. Bai, and B. Chen, "Existence for fractional Dirichlet boundary value problem under barrier strip conditions," The Journal of Nonlinear Sciences and Applications, vol. 10, no. 7, pp. 3592-3598, 2017.

[34] E. Zeidler, Nonlinear Tunctional Analysis and its Applications I: Fixed-Point Theorems, Springer-Verlag, New York, NY, USA, 1986. 